\title{
Electron-phonon interaction in bulk Pb: Beyond the Fermi surface
}

\author{
I. Yu. Sklyadneva, ${ }^{1,2,3,4}$ R. Heid, ${ }^{1}$ P. M. Echenique, ${ }^{2,5,6}$ K.-B. Bohnen, ${ }^{1}$ and E. V. Chulkov ${ }^{2,5,6}$ \\ ${ }^{1}$ Karlsruher Institut für Technologie, Institut für Festkörperphysik, D-76021 Karlsruhe, Germany \\ ${ }^{2}$ Donostia International Physics Center (DIPC), 20018 San Sebastián/Donostia, Basque Country, Spain \\ ${ }^{3}$ Institute of Strength Physics and Materials Science, SB RAS, 634021, Tomsk, Russia \\ ${ }^{4}$ Tomsk State University, 6340501, Tomsk, Russia \\ ${ }^{5}$ Departamento de Física de Materiales, Facultad de Ciencias Químicas, UPVIEHU, Apartado 1072, 20080 San Sebastián/Donostia, \\ Basque Country, Spain \\ ${ }^{6}$ Centro de Física de Materiales CFM - Materials Physics Center MPC, Centro Mixto CSIC-UPV/EHU, 20018 San Sebastian/Donostia, Spain
}

(Received 31 January 2012; published 6 April 2012)

\begin{abstract}
The electron-phonon interaction in bulk $\mathrm{Pb}$ and the influence of spin-orbit coupling (SOC) on the pairing strength are investigated within the density-functional theory and linear-response approach in the mixed-basis pseudopotential representation. We find that the SOC-induced modifications of both electronic bands and lattice vibrations result in striking changes in the phonon-induced scattering of excited electrons (holes). The strength of the momentum-averaged electron-phonon interaction $\lambda(E)$ ranges from 0 to 2.5 while the contribution to $\lambda(E)$ from particular electronic states can even exceed 4. It is found that the coupling strength is exceedingly strong for phonon-induced transitions between electronic states of $p$ and $d$ symmetry. The electron-phonon coupling in $\mathrm{Pb}$ also shows a band dependence which, in particular, results in two different sets of electron-phonon coupling parameters on the two sheets of the Fermi surface.
\end{abstract}

DOI: 10.1103/PhysRevB.85.155115

PACS number(s): 63.20.kd

\section{INTRODUCTION}

The electron-phonon ( $e$-ph) interaction in bulk metals and at metal surfaces is one of fundamental many-body processes which can be tested both experimentally and theoretically. ${ }^{1-6}$ In particular, the $e$-ph coupling affects the lifetime and the dispersion of excited electrons (or holes). ${ }^{2,7,8}$ The lifetime or the linewidth (inverse lifetime) is an essential quantity, describing the decay of electron (hole) excitations ${ }^{7,9}$ which are important in many processes such as photochemical and catalytical reactions. ${ }^{10,11}$

Lead as a strong-coupling superconductor has been a focus of various theoretical studies of electron-phonon coupling properties, ${ }^{6,12-21}$ and a very detailed information concerning the $e$-ph interaction at the Fermi level $\left(E_{\mathrm{F}}\right)$ is now available. ${ }^{13,14,16-19,21}$ Recent $a b$ initio local-density calculations $^{21}$ have shown that the strength of electronphonon interaction, $\lambda\left(E_{\mathrm{F}}\right)$, is strongly influenced by spin-orbit coupling (SOC). The calculations including SOC revealed that relativistic corrections increase $\lambda\left(E_{\mathrm{F}}\right)$ by $44 \%$ from 1.08 up to 1.56 , solving the long-standing contradiction between too small values of $\lambda\left(E_{\mathrm{F}}\right)$ obtained in previous first-principles calculations ${ }^{16,18,19}$ and the experimental $e$ ph coupling parameter, ${ }^{14} \lambda\left(E_{\mathrm{F}}\right)=1.55$. The origin of the SOC-induced enhancement lies both in a softening of the phonon spectrum and in an increase in the $e$-ph coupling matrix elements. ${ }^{21}$ Theoretical evaluations ${ }^{18}$ have also shown the band and momentum anisotropy of the electron-phonon interaction on the Fermi surface of $\mathrm{Pb}$ that has been connected with the different orbital character of the bands crossing the Fermi level.

Here we analyze the electron-phonon interaction as well as the SOC effect on the $e$-ph coupling strength in electronic states situated away from the Fermi energy. On the one hand, this allows us to calculate the electron-phonon coupling parameter averaged over electron momentum, $\lambda(E)$, at $E \neq E_{\mathrm{F}}$ and to estimate the effect of variations in the electronic density of states, $N(E)$, on $\lambda(E)$. This relationship has been probed experimentally by doping of $\mathrm{Pb}$ with $\mathrm{Tl}$ and $\mathrm{Bi}$ to change the valence electron content. ${ }^{14}$ From tunneling measurements various superconducting properties of the respective alloys were determined as a function of doping. On the other hand, one can evaluate the influence of spin-orbit coupling on the pairing strength of particular electron (hole) states in the phonon-induced scattering processes and calculate the electron-phonon contribution to the lifetime (linewidth) of excited electrons. The present study addresses the $e$-ph coupling properties of electron and hole states in the energy interval from $-4 \mathrm{eV}$ to $+10 \mathrm{eV}$ with respect to $E_{\mathrm{F}}$ performing both scalar relativistic calculations and calculations including SOC within density-functional theory.

The paper is organized as follows. In Sec. II a short outline of the calculation method is given. Section III contains the results for the electron-phonon coupling. Finally, in Sec. IV the conclusions are drawn.

\section{CALCUlation DETAILS}

The strength of the electron-phonon interaction for an electron (hole) state of a given momentum $\mathbf{k}$ and band index $i$ is described by a dimensionless coupling constant ${ }^{1} \lambda_{\mathbf{k} i}$ :

$$
\lambda_{\mathbf{k} i}=2 \int_{0}^{\omega_{\max }} \frac{\alpha^{2} F_{\mathbf{k} i}(\omega)}{\omega} d \omega,
$$

where $\omega_{\max }$ is the maximum phonon frequency. The corresponding state dependent Eliashberg function $\alpha^{2} F_{\mathbf{k} i}(\omega)$, which gives the effectiveness of a phonon with energy $\hbar \omega$ to scatter electrons, is given by

$$
\alpha^{2} F_{\mathbf{k} i}(\omega)=\sum_{\mathbf{q}, v, f} \delta\left(\epsilon_{\mathbf{k}+\mathbf{q} f}-\epsilon_{\mathbf{k} i}\right)\left|g_{\mathbf{k}+\mathbf{q} f, \mathbf{k} i}^{\mathbf{q} v}\right|^{2} \delta\left(\omega-\omega_{\mathbf{q} \nu}\right) .
$$


Here $\epsilon_{\mathbf{k} i}$ and $\epsilon_{\mathbf{k}+\mathbf{q} f}$ are the energies of the initial and final electronic states, respectively, and the so-called quasielastic approximation is used: $\delta\left(\epsilon_{\mathbf{k} i}-\epsilon_{\mathbf{k}+\mathbf{q} f} \mp \omega_{\mathbf{q}, v}\right) \approx \delta\left(\epsilon_{\mathbf{k} i}-\epsilon_{\mathbf{k}+\mathbf{q} f}\right)$. The $e$-ph matrix element $g_{\mathbf{k}+\mathbf{q} f, \mathbf{k} i}^{\mathbf{q} v}$ indicates the probability of scattering from the state $(\mathbf{k} i)$ to a final state $(\mathbf{k}+\mathbf{q} f)$ by a phonon $(\mathbf{q} v)$ with frequency $\omega_{\mathbf{q} v}$.

When $\lambda_{\mathbf{k} i}$ is averaged over electron momentum at a fixed energy $\epsilon_{\mathbf{k} i}=E$ one obtains the energy resolved $e$-ph coupling constant $^{22} \lambda(E)$ :

$$
\lambda(E)=2 \int_{0}^{\omega_{\max }} \frac{\alpha^{2} F(E ; \omega)}{\omega} d \omega,
$$

where the corresponding energy resolved Eliashberg spectral function is given by

$$
\begin{aligned}
\alpha^{2} F(E ; \omega)= & \frac{1}{N(E)} \sum_{\mathbf{k} i} \alpha^{2} F_{\mathbf{k} i}(\omega) \delta\left(\epsilon_{\mathbf{k} i}-E\right) \\
= & \frac{1}{N(E)} \sum_{\mathbf{q} v} \delta\left(\omega-\omega_{\mathbf{q} v}\right) \sum_{\mathbf{k}, i, f}\left|g_{\mathbf{k}+\mathbf{q} f, \mathbf{k} i}^{\mathbf{q} v}\right|^{2} \\
& \times \delta\left(\epsilon_{\mathbf{k} i}-E\right) \delta\left(\epsilon_{\mathbf{k}+\mathbf{q} f}-E\right) .
\end{aligned}
$$

Here $N(E)=\sum_{\mathbf{k} i} \delta\left(\epsilon_{\mathbf{k} i}-E\right)$ is the density of electronic states at energy $E$.

Both scalar relativistic calculations and calculations including SOC were performed within density-functional theory and local-density approximation for the exchange-correlation energy. ${ }^{23}$ Electron-phonon coupling properties were calculated using the linear-response technique ${ }^{24,25}$ as implemented in the mixed-basis pseudopotential method, ${ }^{26,27}$ which employs a combination of local functions and plane waves for the representation of valence states. ${ }^{28}$ We included $5 d$ semicore states in the construction of norm-conserving scalar and fully relativistic pseudopotentials. ${ }^{29}$ To treat the deep $d$ potentials one $d$-type local function at each atomic site of $\mathrm{Pb}$ is added to accelerate the convergence and to reduce the size of the plane-wave basis set for which a cutoff of $20 \mathrm{Ry}$ is found to be enough. Further details of the spin-orbit coupling implementation within the mixed-basis pseudopotential method can be found in the previous paper. ${ }^{21}$ The Brillouin zone (BZ) integrations in Eq. (4) were performed by sampling a dense $32 \times 32 \times 32$ k-point mesh $^{30}$ corresponding to 2992 points in the irreducible wedge of the BZ. The $\delta$ functions were replaced by the Gaussian functions with the width of $0.2 \mathrm{eV}$.

\section{RESULTS AND DISCUSSION}

We have calculated the electron-phonon coupling parameter $\lambda_{\mathbf{k} i}$ as a function of momentum for three $p$-like electronic bands along high-symmetry directions: $Z, \Sigma$, and $\Lambda$. The bands are marked by $B_{7}, B_{8}$, and $B_{9}$ in Fig. 1 . The radii of circles in the figure are proportional to the corresponding value of $\lambda_{\mathbf{k} i}$. The largest couplings are found for electronic states in the $B_{9}$ band at $\epsilon_{\mathbf{k} i} \geqslant 6.5 \mathrm{meV}$ where phonon-induced transitions between $p$ - and $d$-like bands (the latter are shown in Fig. 1 by thick lines) become actively involved in the scattering processes. The strength of $e$-ph coupling for the electronic states in band $B_{8}$ (at these energies) is markedly smaller because, in this case, the scattering to $d$-like states is less effective and a larger contribution to $\lambda_{\mathbf{k} i}$ comes from

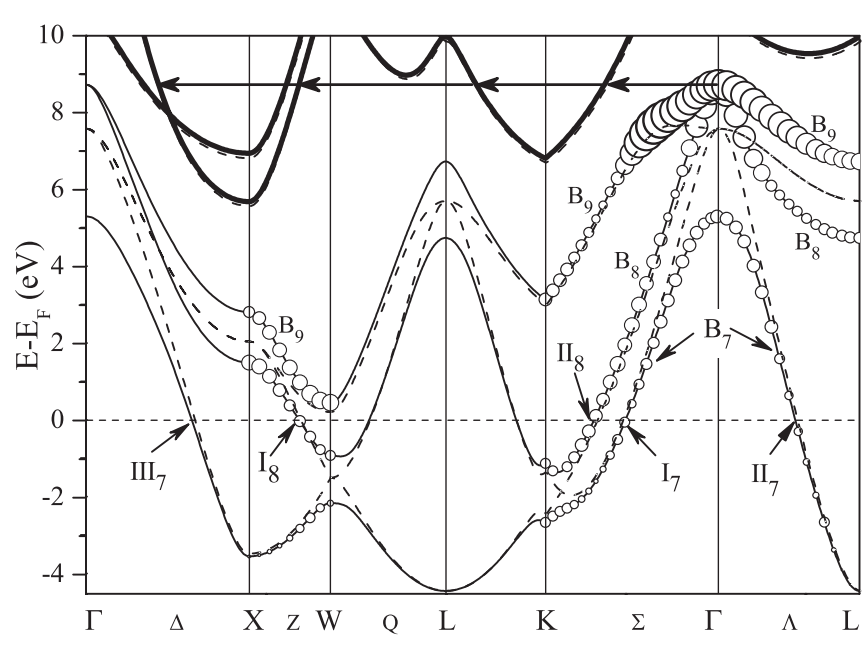

FIG. 1. Electronic band structure of bulk $\mathrm{Pb}$. The solid and dashed dispersion lines correspond to calculations with and without SOC, respectively. Electronic states of $d$ symmetry are displayed by thick lines. The radii of circles are proportional to the strength of the $e$-ph interaction $\lambda_{\mathbf{k} i}$ (including SOC) shown for electronic states along the $Z, \Sigma$, and $\Lambda$ symmetry directions. Arrows indicate those electronic transitions which predominantly contribute to $\lambda_{\mathbf{k} i}$ of the electronic state at $\Gamma(\epsilon=8.73 \mathrm{eV})$.

$B_{8} \rightarrow B_{9}$ transitions. The coupling between $p$-like electronic bands is found to be considerably weaker as compared to the $e$-ph matrix elements for $p$ - $d$ pairs.

The influence of spin-orbit coupling on the strength of the $e$-ph interaction is shown in Fig. 2 for energy bands $B_{7}$ and $B_{8}$ along the $\Sigma$ and $\Lambda$ symmetry directions. At the $\Gamma$ point the bands are degenerate at $\epsilon=7.58 \mathrm{eV}$ in the case of the semirelativistic calculation, but split by the SOC into two states at 5.3 and $8.73 \mathrm{eV}$, respectively. The SOC-induced shift of the $B_{7}$ band (Fig. 2, $B_{7}$ ) to smaller energies leads to the disappearance of the peak in $\lambda_{\mathbf{k} i}$ around the $\Gamma$ point where the $e$-ph coupling strength decreases from 3.35 (without SOC) to 1.74 (with SOC). It is found that the peak is caused by transitions from the $p$-like $B_{7}$ band to $d$-like electronic bands whose contribution to $\lambda_{\mathbf{k} i}$ turns out to be exceedingly strong. Beyond the energy region where the $p-d$ transitions are possible the $e$-ph coupling parameter for electronic states varies by a small amount around an averaged value of $\lambda_{\mathbf{k} i} \approx 1.8$ (with SOC) slightly decreasing on approaching the Fermi level. Similarly, the phonon-mediated coupling of electronic states in the upper energy band (Fig. 2, $B_{8}$ ) is, in general, stronger at $\epsilon_{\mathbf{k} i} \geqslant 6.5 \mathrm{eV}$ due to the transitions to $d$-like electronic states, especially to those which are partly or entirely of $d_{x^{2}-y^{2}}$ or $d_{3 z-r^{2}}$ symmetry (arrows in Fig. 1). The contribution of the $p-d$ transitions to the strength of the $e$-ph interaction is indicated by hatched areas in Fig. 2 .

The values of $\lambda_{\mathbf{k}_{\mathrm{F}}}$ for some electronic states at $E_{\mathrm{F}}$ are shown in Table I. In Fig. 1 the states are marked as $\mathrm{I}_{7}, \mathrm{II}_{7}, \mathrm{III}_{7}, \mathrm{I}_{8}$, and $\mathrm{II}_{8}$. At the Fermi energy, the strength of the $e$-ph interaction in band $B_{7}$ is found to be $14-17 \%$ (with SOC) smaller than the value averaged over all possible $e$-ph scattering events on the Fermi surface, ${ }^{21} \lambda\left(E_{\mathrm{F}}\right)=1.56$. The contribution to $\lambda\left(E_{\mathrm{F}}\right)$ of the upper energy band, $B_{8}$, is larger, e.g., 1.79 for the electronic state $\mathrm{II}_{8}$ in the $\Sigma$ symmetry direction. On average, 

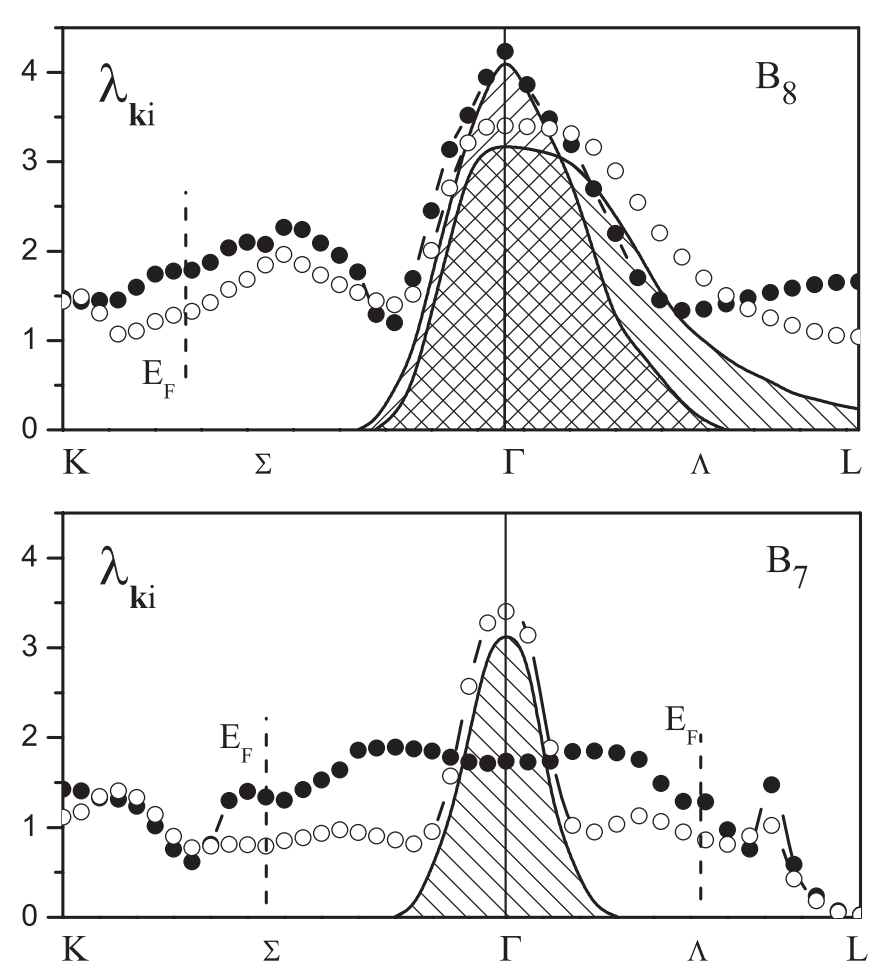

FIG. 2. Electron-phonon coupling parameter $\lambda_{\mathbf{k} i}$ as a function of momentum for two $p$-like electron (hole) bands along the $\Sigma$ and $\Lambda$ symmetry directions. The bands are marked by $B_{7}$ and $B_{8}$ in Fig. 1. The full and open circles correspond to the calculations with and without SOC, respectively. The contribution to the $e$-ph coupling coming from the scattering with electronic states of $d$ type is indicated by hatched areas for band $B_{8}$ (both with and without SOC) and band $B_{7}$ (without SOC).

the strength of $e$-ph interaction in band $B_{8}$ is $\sim 1.3$ times higher than for $B_{7}$. That leads to two different sets of $\lambda_{\mathbf{k} i}$ for the two Fermi-surface sheets. As discussed before, ${ }^{18}$ the sheet derived from band $B_{7}$ has an octahedral shape, while the other possesses a tubularlike structure (from band $B_{8}$ ). The strength of the electron-phonon interaction in the tubularlike sheet is about 1.6-1.8, but it is only about $1.3-1.5$ for the octahedral sheet. It should be noted that the difference between the two sets is not so large as that found in another two-band superconductor, $\mathrm{MgB}_{2}$, where the coupling in one electronic band crossing $E_{\mathrm{F}}$ is three times larger than in the other. ${ }^{31}$ In $\mathrm{Pb}$, the band dependence of the $e$-ph interaction can be related to a slightly different orbital character of the electronic bands. While the lower band, $B_{7}$, has a mixed $s-p$ character, the upper one, $B_{8}$, is more $p$ - $d$ like. Similar data for bulk Pb were reported in Ref. 18 where semirelativistic theoretical estimates also showed the band anisotropy of the $e$-ph interaction on

TABLE I. Electron-phonon coupling parameters for electronic bands $B_{7}$ and $B_{8}$ at the Fermi level (see Fig. 1).

\begin{tabular}{lccccc}
\hline \hline$\lambda_{\mathbf{k}_{\mathrm{F}}}$ & $\mathrm{I}_{8}$ & $\mathrm{II}_{8}$ & $\mathrm{I}_{7}$ & $\mathrm{II}_{7}$ & $\mathrm{III}_{7}$ \\
\hline Without SOC & 1.15 & 1.33 & 0.79 & 0.84 & 0.82 \\
With SOC & 1.58 & 1.79 & 1.34 & 1.30 & 1.50 \\
\hline \hline
\end{tabular}

the Fermi surface. It was suggested ${ }^{18}$ that the more localized electronic states are more coupled to the lattice and, thereby, produce a higher value of $\lambda$. As is evident from Table I, the spin-orbit interaction enhances the $e$-ph coupling in electronic states on the Fermi surface. However, the SOC effect on the strength of the $e$-ph interaction is clearly band dependent. The SOC influence is particularly strong for band $B_{7}$ where $\lambda_{\mathbf{k}_{\mathrm{F}}}$ increases $\sim 1.55-1.8$ times (depending on the crystal direction) with SOC corrections included.

As is known the electron-phonon interaction changes the dispersion and influences the lifetime of electronic states. The effect can be expressed in terms of the complex $e$-ph self-energy $\Sigma$, where the real part allows us to evaluate the shift in electronic energies while the lifetime describing the decay of excited electrons or holes can be obtained from the imaginary part. The latter can be written in terms of the Eliashberg function as ${ }^{32}$

$$
\begin{aligned}
\operatorname{Im} \Sigma_{\mathbf{k} i}(\epsilon)= & -\pi \int_{0}^{\omega_{\max }} d \omega \alpha^{2} F_{\mathbf{k} i}(\omega) \\
& \times\left[2 n(\omega)+f\left(\epsilon_{\omega+\mathbf{k} i}\right)+f\left(\epsilon_{\omega-\mathbf{k} i}\right)\right]
\end{aligned}
$$
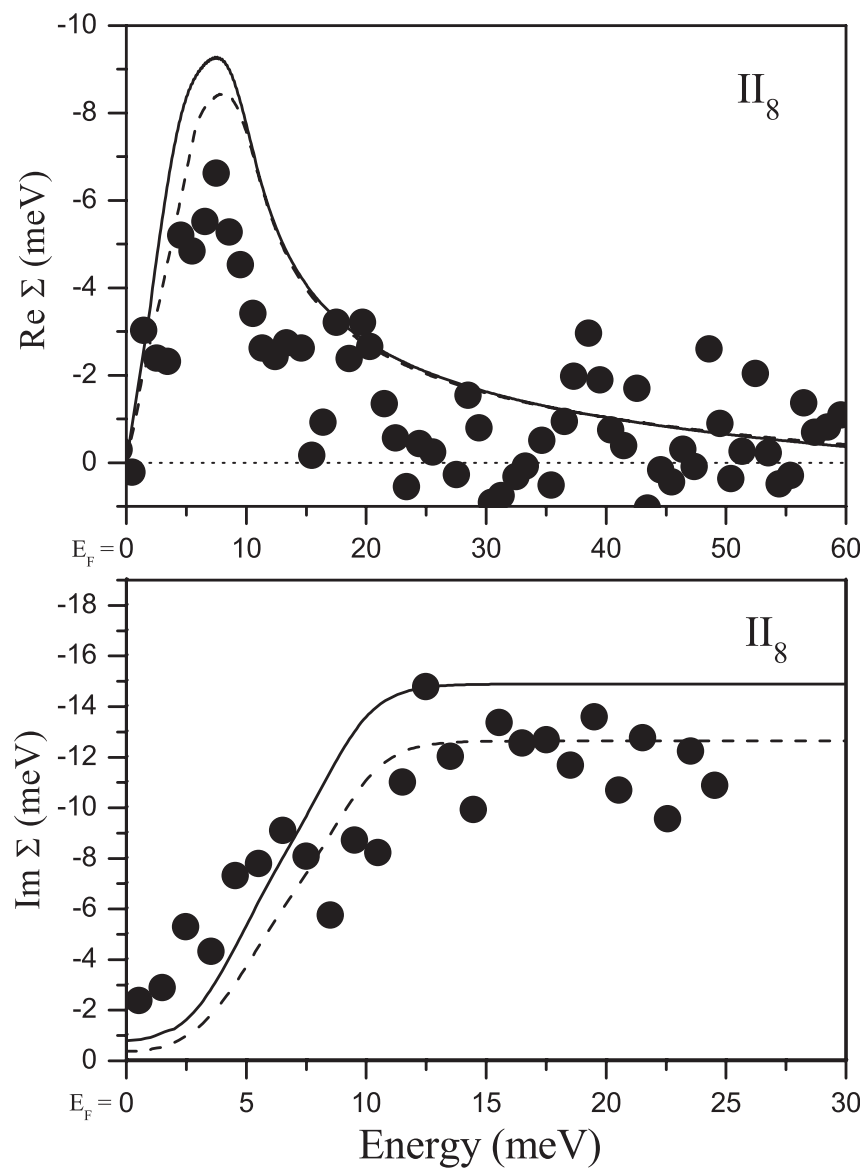

FIG. 3. Real and imaginary parts of the electron-phonon selfenergy $\Sigma$ for electronic state $\mathrm{II}_{8}$ (see Fig. $1, \Gamma K$ symmetry direction). The solid (dashed) lines correspond to calculations with (without) SOC. The imaginary part is shifted vertically to match the experimental data (full circles) taken from Ref. 17. 


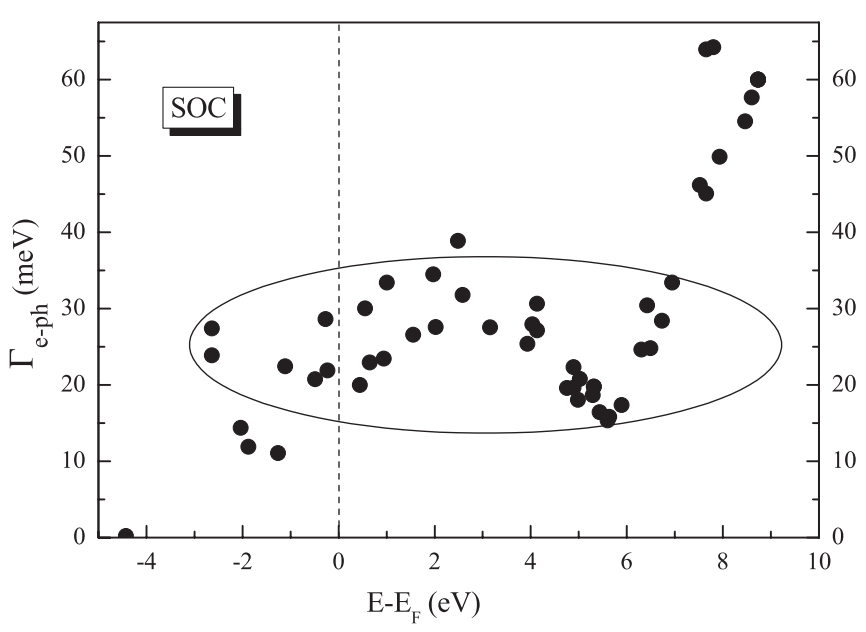

FIG. 4. Calculated electron-phonon contribution to the linewidth as a function of energy for excited electrons and holes in the $B_{7}$, $B_{8}$, and $B_{9}$ bands (see Fig. 1) at k's along the $\Sigma$ and $\Lambda$ symmetry directions $(T=0 \mathrm{~K})$.

while the real part is conveniently calculated using the Kramers-Kronig transformation

$$
\operatorname{Re} \Sigma_{\mathbf{k} i}(\epsilon)=\frac{1}{\pi} \mathcal{P} \int_{-\infty}^{\infty} d \xi \frac{\operatorname{Im} \Sigma_{\mathbf{k} i}(\xi)}{\xi-\epsilon} .
$$

Here, $f$ and $n$ are the Fermi and Bose distribution functions, respectively. Figure 3 shows the real and imaginary parts of $e$-ph self-energy $\Sigma$ for the $\mathrm{II}_{8}$ electronic state of band $B_{8}$, which lies at the Fermi-level crossing along the $\Sigma$ direction (on the $\Gamma K$ line). To demonstrate the influence of spin-orbit interaction on the electron-phonon coupling data both with and without SOC are presented. Also shown are the experimental values obtained on a $\mathrm{Pb}(110)$ surface at $T=8 \mathrm{~K}$ for a state of band $B_{8}$ at the Fermi energy, which was actually measured slightly off the high-symmetry $\Sigma$ direction toward $\Gamma X .{ }^{17}$ Apart from the difference in maximum height, the agreement between experimental and theoretical data is gratifying, in particular, with respect to the position of the Re $\Sigma$ maximum. In the limit $T \rightarrow 0$, the slope of $\operatorname{Re} \Sigma(\epsilon)$ at $\epsilon=E_{\mathrm{F}}$ determines the $e$-ph coupling strength $\lambda_{\mathbf{k}_{\mathrm{F}}}$. The value obtained in the present calculation, $\lambda_{\mathbf{k}_{\mathrm{F}}}=1.79$, is slightly larger than the one deduced from experiment $\lambda_{\mathbf{k}_{\mathrm{F}}}=1.55 .{ }^{17}$ This is a reasonable agreement in view of the fact that we are comparing two distinct states, and that a precise experimental determination of the slope is usually complicated by the finite instrumental resolution ${ }^{33}$ that tends to smear out the band renormalization, in particular near $E_{\mathrm{F}}$.

Figure 4 shows the calculated electron-phonon contribution to the linewidth, $\Gamma_{e-\mathrm{ph}}=-2 \operatorname{Im} \Sigma_{\mathbf{k} i}\left(\epsilon_{\mathbf{k} i}\right)$, of excited electrons (holes) in the $p$-like $B_{7}, B_{8}$, and $B_{9}$ bands at momenta along the $\Sigma$ and $\Lambda$ symmetry directions. The data were obtained for $T=0 \mathrm{~K}$. Evidently, the values of $\Gamma_{e \text {-ph }}$ are not large and usually alter between 15 and $35 \mathrm{meV}$. Larger values of $\Gamma_{e \text {-ph }}$ are obtained for excited electrons with $\epsilon_{\mathbf{k} i} \geqslant 7 \mathrm{eV}$ in the $B_{8}$ and $B_{9}$ bands at $\mathbf{k}$ 's around the BZ center where the contribution of transitions from $p$ - to $d$-like electronic bands becomes dominant. Very recently, the contribution of electron-electron inelastic-scattering processes to the decay of electron excitations, $\Gamma_{e-e}$, was studied for bulk $\mathrm{Pb}$ by
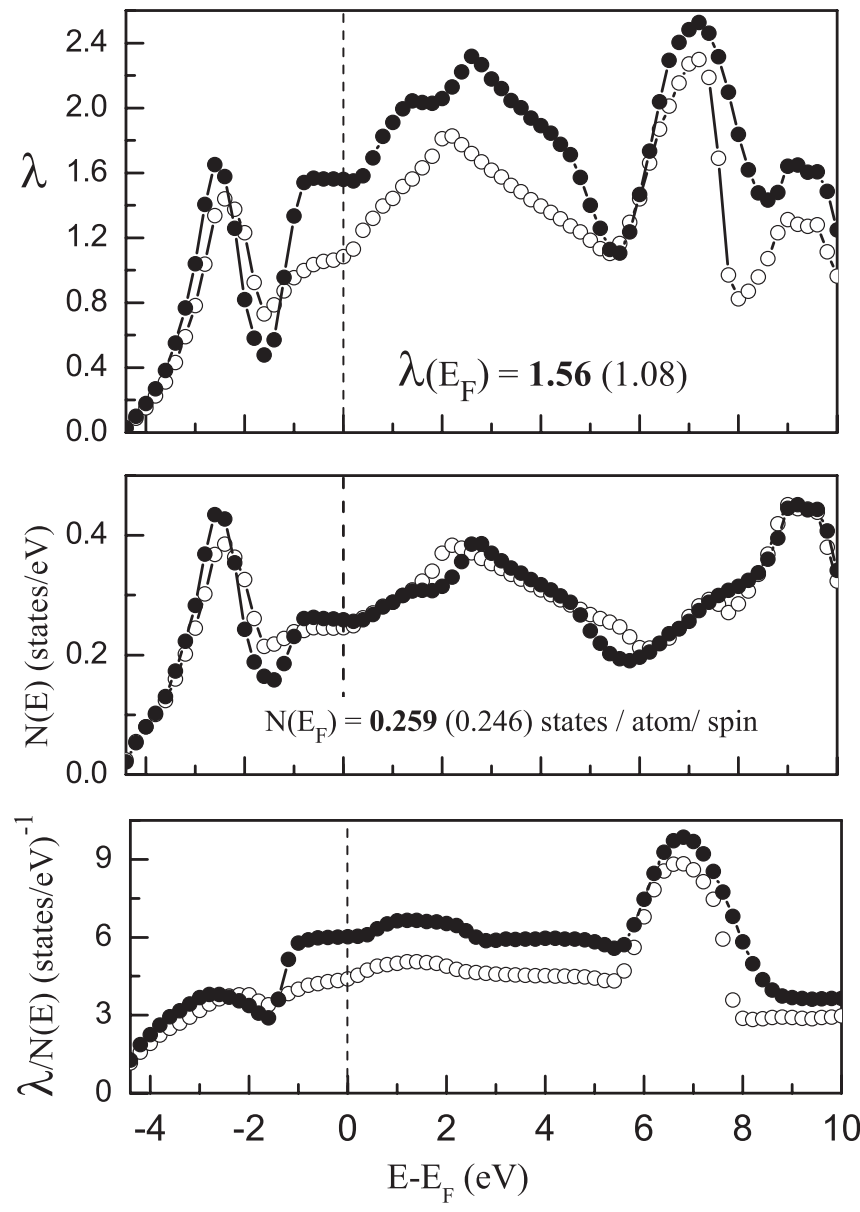

FIG. 5. Electron-phonon coupling parameter $\lambda(E)$ averaged over electron momentum (the upper panel), electronic density of states $N(E)$ (the middle panel), and $\lambda(E) / N(E)$ (the lower panel) as functions of energy. The open circles denote semirelativistic calculations while the full ones represent calculations including spin-orbit interaction.

means of first-principles calculations for excited electrons and holes with energies up to $8 \mathrm{eV}^{34}$ Both band-structure effects as well as the influence of spin-orbit interaction have been analyzed. The values of $\Gamma_{e-e}$ for the $p$ states at $\epsilon_{\mathrm{k} i} \leqslant 8 \mathrm{eV}$ vary from 0 (at $E_{\mathrm{F}}$ ) to $1400 \mathrm{meV}$ showing a parabolic distribution with energy. ${ }^{34}$ Thus the phonon-induced contribution to the linewidth of electron excitations is usually an order of magnitude smaller than the contribution of the electron-electron inelastic scattering. The only exception is the vicinity of the Fermi energy $\left(\left|\epsilon_{\mathbf{k} i}-E_{\mathrm{F}}\right| \leqslant 2 \mathrm{eV}\right)$ where $\Gamma_{e-\mathrm{ph}}$ is of the same order of magnitude and even exceeds $\Gamma_{e-e}$ at $\epsilon_{\mathbf{k} i} \rightarrow E_{\mathrm{F}}$.

Next we have determined the momentum-averaged coupling constant $\lambda(E)$ at various energies both below and above $E_{\mathrm{F}}(-4.4 \mathrm{eV} \leqslant E \leqslant 10 \mathrm{eV})$. Figure 5 shows the strength of the $e$-ph coupling as well as the density of electronic states, $N(E)$, calculated both with and without SOC. The major factors which determine $\lambda(E)$ are the number of electronic states available for scattering processes (phase space) and the $e$-ph matrix elements. From the ratio $\lambda(E) / N(E)$ shown in the lower panel of Fig. 5, it is obvious that in the range $-1 \mathrm{eV} \leqslant$ $E \leqslant 6 \mathrm{eV}, \lambda(E)$ is essentially dominated by variations in the 
phase space, while matrix elements are approximately energy independent. This trend was also inferred from electrontunneling measurements on $\mathrm{Pb}$ doped by $\mathrm{Tl}$ (hole doping) and $\mathrm{Bi}$ (electron doping). ${ }^{14}$ For $E<-1 \mathrm{eV}$, one observes a drop of the ratio $\lambda(E) / N(E)$ indicating a reduction of the $e$-ph matrix elements. In addition, the strong peak in $\lambda(E)$ at $6 \mathrm{eV} \leqslant E \leqslant 8 \mathrm{eV}$, has no counterpart in $N(E)$, but reflects a strong increase in the related phonon-mediated electronic transitions, which involve primarily $p$ - $d$ transitions with rather large matrix elements.

The influence of SOC on the $e$-ph interaction is rather intricate. As it was shown in the previous paper ${ }^{21}$ there are many factors which interfere. The inclusion of SOC induces a softening of practically all phonon branches. ${ }^{21,35}$ The shift to lower frequencies promotes an increase of $\lambda$. The SOC also makes changes in the electronic band structure of $\mathrm{Pb}$ (see, for example, Fig. 1 and Refs. 21, 36, and 37). On the one hand, the density of electronic states changes (see, Fig. 5, the middle panel). The largest variations in $N(E)$ are related to the SOC-induced splitting of electronic bands at some high-symmetry points. Such splitting leads to changes of more than $20 \%$ (at $\sim-2,-1.5,2$, and $5.5 \mathrm{eV}$ corresponding to the $K, W, X$, and $L$ symmetry points, respectively). However, at the Fermi energy the modifications are minor: $N(E)$ increases only by $6 \%$. On the other hand, the structure of $p$-like electronic bands changes considerably. That modifies the phonon-induced electronic transitions and, thereby, can affect deeply $e$-ph matrix elements. For example, at $E=$ $+2 \mathrm{eV} \lambda$ increases by $14 \%$ in spite of the fact that $N(E)$ becomes smaller.

\section{SUMMARY}

A first-principles study of the influence of spin-orbit interaction on the electron-phonon coupling in electron and hole states of bulk $\mathrm{Pb}$ has been presented. It is found that the strength of the $e$-ph interaction averaged over electron momentum $\lambda(E)$ ranges from 0 to 2.5 while for particular electronic states $\lambda_{\mathbf{k} i}$ can even exceed 4 . In a region around the Fermi energy, the variations of $\lambda(E)$ are controlled by the available phase space for scattering processes. At higher energies, the appearance of electronic states with $d$ symmetry promotes very strong $e$-ph coupling between $p$ and $d$ states which results in an enhancement of the corresponding momentum averaged value of $\lambda(E)$. The electron-phonon coupling also shows a band dependence. In particular, the band anisotropy results in two different sets of $\lambda_{\mathbf{k}_{\mathrm{F}}}$ related to a slightly different orbital character of the two electronic bands crossing $E_{\mathrm{F}}$. On the average, the strength of the $e$-ph interaction in the tubularlike sheet is $\sim 1.3$ times higher than in the octahedral one.

The influence of spin-orbit interaction on phonon-mediated electronic transitions is largely determined by the SOCinduced variation of the $e$-ph coupling matrix elements. The modifications in the phase space available for scattering is only of minor importance. The SOC effect is also found to be band dependent. In particular, on the Fermi surface, the largest relativistic corrections are found for electronic states on the octahedral sheet. The large differences between calculations with and without SOC indicate that spin-orbit interaction must be taken into account for a proper quantitative determination of both state dependent and momentum averaged electronphonon coupling quantities of bulk $\mathrm{Pb}$.

\section{ACKNOWLEDGMENTS}

We acknowledge financial support of the University of the Basque Country UPV/EHU (Grant No. GIC07-IT-36607), the Departamento de Educación del Gobierno Vasco, and the Spanish Ministerio de Ciencia e Innovación (Grant No. FIS2010-19609-C02-01).
${ }^{1}$ G. Grimvall, The Electron-Phonon Interaction in Metals in Selected Topics in Solid State Physics, edited by E. Wohlfarth, Vol. 16 (North-Holland, New York, 1981).

${ }^{2} \mathrm{Ph}$. Hofmann, I. Yu. Sklyadneva, E. D. L. Rienks, and E. V. Chulkov, New J. Phys. 11, 125005 (2009).

${ }^{3}$ J. Kroger, Rep. Prog. Phys. 69, 899 (2006).

${ }^{4}$ E. W. Plummer, J. Shi, S.-J. Tang, E. Rotenberg, and S. D. Kevan, Prog. Surf. Sci. 74, 251 (2003).

${ }^{5}$ S. Mathias, S. V. Eremeev, E. V. Chulkov, M. Aeschlimann, and M. Bauer, Phys. Rev. Lett. 103, 026802 (2009).

${ }^{6}$ I. Yu. Sklyadneva, G. Benedek, E. V. Chulkov, P. M. Echenique, R. Heid, K.-P. Bohnen, and J. P. Toennies, Phys. Rev. Lett. 107, 095502 (2011).

${ }^{7}$ E. V. Chulkov, A. G. Borisov, J. P. Gauyacq, D. Sánchez-Portal, V. M. Silkin, V. P. Zhukov, and P. M. Echenique, Chem. Rev. 106, 4160 (2006).

${ }^{8}$ A. Eiguren, B. Hellsing, E. V. Chulkov, and P. M. Echenique, Phys. Rev. B 67, 235423 (2003).

${ }^{9}$ I. Po Hong, C. Brun, F. Patthey, I. Y. Sklyadneva, X. Zubizarreta, R. Heid, V. M. Silkin, P. M. Echenique, K. P. Bohnen, E. V. Chulkov, and W. D. Schneider, Phys. Rev. B 80, 081409(R) (2009)
${ }^{10}$ K. Watanabe, D. Menzel, N. Nilius, and H.-J. Freud, Chem. Rev. 106, 4301 (2006).

${ }^{11}$ P. Saalfrank, Chem. Rev. 106, 4116 (2006).

${ }^{12}$ J. R. Schrieffer, D. J. Scalapino, and J. W. Wilkins, Phys. Rev. Lett. 10, 336 (1963)

${ }^{13}$ W. L. McMillan and J. M. Rowell, in Superconductivity, edited by R. D. Parks (Marcel Dekker, New York, 1969), Vol. 1.

${ }^{14}$ R. C. Dynes and J. M. Rowell, Phys. Rev. B 11, 1884 (1975).

${ }^{15}$ S. Y. Savrasov and D. Y. Savrasov, Phys. Rev. B 54, 16487 (1996).

${ }^{16}$ A. Y. Liu and A. A. Quong, Phys. Rev. B 53, R7575 (1996).

${ }^{17}$ F. Reinert, B. Eltner, G. Nicolay, D. Ehm, S. Schmidt, and S. Hüfner, Phys. Rev. Lett. 91, 186406 (2003); Phys. Rev. Lett. 92, 089904(E) (2004).

${ }^{18}$ A. Floris, A. Sanna, S. Massidda, and E. K. U. Gross, Phys. Rev. B 75, 054508 (2007).

${ }^{19}$ P. Aynajian, T. Keller, L. Boeri, S. M. Shapiro, K. Habicht, and B. Keimer, Science 319, 1509 (2008).

${ }^{20}$ C. Brun, I. Po Hong, F. Patthey, I. Y. Sklyadneva, R. Heid, P. M. Echenique, K. P. Bohnen, E. V. Chulkov, and W. D. Schneider, Phys. Rev. Lett. 102, 207002 (2009).

${ }^{21}$ R. Heid, K.-P. Bohnen, I. Yu. Sklyadneva, and E. V. Chulkov, Phys. Rev. B 81, 174527 (2010). 
${ }^{22}$ P. B. Allen and M. L. Cohen, Phys. Rev. 187, 525 (1969).

${ }^{23}$ L. Hedin and B. I. Lundqwist, J. Phys. C 4, 2064 (1971).

${ }^{24}$ N. E. Zein, Sov. Phys. Solid State 26, 1825 (1984) [Fiz. Tverd. Tela (Leningrad) 26, 3028 (1984)].

${ }^{25}$ S. Baroni, S. de Gironcoli, A. Dal Corso, and P. Giannozzi, Rev. Mod. Phys. 73, 515 (2001).

${ }^{26}$ R. Heid and K.-P. Bohnen, Phys. Rev. B 60, R3709 (1999).

${ }^{27}$ B. Meyer, C. Elsässer, F. Lechermann, and M. Fähnle, FORTRAN90, Program for Mixed-Basis-Pseudopotential Calculations for Crystals (Max-Planck-Institut für Metallforschung, Stuttgart).

${ }^{28}$ S. G. Louie, K.-M. Ho, and M. L. Cohen, Phys. Rev. B 19, 1774 (1979).

${ }^{29}$ D. Vanderbilt, Phys. Rev. B 32, 8412 (1985).
${ }^{30}$ H. J. Monkhorst and J. D. Pack, Phys. Rev. B 13, 5188 (1976).

${ }^{31}$ H. J. Choi, D. Roundy, H. Sun, M. L. Cohen, and S. G. Louie, Phys. Rev. B 66, 020513(R) (2002).

${ }^{32}$ G. D. Mahan, Many-Particle Physics (Plenum, New York, 1990).

${ }^{33}$ J. E. Gayone, C. Kirkegaard, J. W. Wells, S. V. Hoffmann, Z. Li, and P. Hofmann, Appl. Phys. A 80, 943 (2005).

${ }^{34}$ X. Zubizarreta, V. M. Silkin, and E. V. Chulkov, Phys. Rev. B 84, 115144 (2011).

${ }^{35}$ A. Dal Corso, J. Phys.: Condens. Matter 20, 445202 (2008).

${ }^{36}$ M. J. Verstraete, M. Torrent, F. Jollet, G. Zérah, and X. Gonze, Phys. Rev. B 78, 045119 (2008).

${ }^{37}$ K. Horn, B. Reihl, A. Zartner, D. E. Eastman, K. Hermann, and J. Noffke, Phys. Rev. B 30, 1711 (1984). 